\begin{tabular}{|c|l|}
\hline Title & Single photon generation from InA IA s single quantum dot \\
\hline Author(s) & $\begin{array}{l}\text { Kimura, S.; Kumano, H.; Endo, M.; Suemune, I.; Y okoi, T.; Sasakura, H.; A dachi, S.; Muto, S.; Song, H. Z; Hirose, S.; } \\
\text { U suki, T. }\end{array}$ \\
\hline Citation & $\begin{array}{l}\text { physica status solidi (c), 2(11), 3833-3837 } \\
\text { https://doi.org/10.1002/pssc.200562027 }\end{array}$ \\
\hline Issue Date & 2005-11-08 \\
\hline Doc URL & http://hdl.handle.net/2115/5601 \\
\hline Rights & Copyright $\odot$ 2005 John Wiley \& Sons, Inc., physica status solidi (c), 2, 11,p3833-3837 \\
\hline Type & article (author version) \\
\hline File Information & PSS2-11.pdf \\
\hline
\end{tabular}

Instructions for use 


\title{
Single-Photon Generation from InAlAs Single Quantum Dot
}

\author{
S. Kimura ${ }^{1 *}$, H. Kumano ${ }^{1}$, M. Endo ${ }^{1}$, I. Suemune ${ }^{1,3}$, T. Yokoi ${ }^{2}$, H. Sasakura ${ }^{3}$,

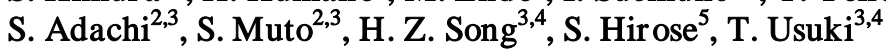 \\ ${ }^{1}$ RIES, Hokkaido Univ. Sapporo 001-0021, Japan \\ ${ }^{2}$ Department of Applied Physics, Hokkaido Univ. Sapporo 060-8628, Japan \\ ${ }^{3}$ CREST JST, Kawaguchi 332-0012, Japan \\ ${ }^{4}$ Fujitsu Ltd., 10-1 Morinosato-Wakamiya, Atsugi 243-0197, Japan \\ ${ }^{5}$ Fujitsu Labs. Ltd., 10-1 Morinosato-Wakamiya, Atsugi 243-0197, Japan
}

Received z z z, revised z z z, accepted z z z

Published online $\mathrm{z} z \mathrm{Z}$

PACS 78.67.Hc, 42.50.Ar, 78.55.Cr, 78.67.-n

Regulated single-photon generation from an InAlAs quantum dot was demonstrated at a wavelength in which Si-avalanche photodiode single-photon detector has high quantum efficiency. Measured excitation power dependence of photon coincidence counts revealed that highly pure single-photon emission was realized at low excitation powers. Beyond a critical excitation, population of multi-exciton states in the quantum dot degraded the purity of single-photon emission.

(C) 2004 WILEY-VCH Verlag GmbH \& Co. KGaA, Weinheim

1 Introduction Quantum cryptography is attracting much more attention recently due to its highly secure nature [1]. Since it is based on physical principles such as indivisibility and non-cloning of single photons [2], eavesdropping will be impossible in principle. At present bit rates in quantum cryptography are limited by both single-photon emitters and detectors. Presently used attenuated laser sources are limited to slow bit rates because of their intrinsic Poisson statistics of emitted photon numbers. So far, single-photon emissions have been achieved by various physical systems to realize alternative singlephoton sources [3]. Among them, semiconductor quantum dots (QDs) are one of the most promising candidates. On the other hand, presently most efficient commercially available single-photon detectors are $\mathrm{Si}$ avalanche photo diodes (Si-APD) and they have high quantum efficiency in the wavelength range of $600 \mathrm{~nm}$ to $800 \mathrm{~nm}$. Realization of single-photon emitters which cover this wavelength range will be promising for achieving overall high-bit-rate quantum cryptography systems.

Previously many groups have studied single-photon emitters, but material systems which match the above wavelength range of $600 \mathrm{~nm}$ to $800 \mathrm{~nm}$ are limited [4-12]. InAlAs QDs are one of the promising candidates for this purpose. However, single-photon emission from InAlAs QDs has not been proved. In this paper, regulated single-photon emission or photon anti-bunching from InAlAs QDs at the wavelength around 770nm will be demonstrated. To realize highly efficient single-photon emitters with ondemand operations, high-probability populations of QD energy states are necessary. Excitation power dependence of the photon anti-bunching properties will be discussed.

2 Experimental A QDs sample was grown on a (001) GaAs substrate by molecular-beam epitaxy. It had two-stacks of QDs layer made of $\operatorname{In}_{0.75} \mathrm{Al}_{0.25} \mathrm{As}$ and $\mathrm{In}_{0.7} \mathrm{Ga}_{0.3}$ As separated with an $\mathrm{Al}_{0.3} \mathrm{Ga}_{0.7} \mathrm{As}$ layer but the coupling effects between the two QDs layers were negligible in this sample. This work was focused to the $\mathrm{In}_{0.75} \mathrm{Al}_{0.25} \mathrm{As}$ QDs layer. These QDs layers were grown in a Stranski-Krastanow mode and were sandwiched by $\mathrm{Al}_{0.3} \mathrm{Ga}_{0.7}$ As layers. The sample surface was covered with a GaAs cap layer. After

\footnotetext{
* Corresponding author: e-mail: skimura@es.hokudai.ac.jp
} 
the growth, mesa structures were formed by electron-beam lithography and wet chemical etching. Further details of this sample were described in Ref. 13.

In our measurements, the sample was kept in a closed-loop He cryostat and kept at $23 \mathrm{~K}$. A He-Ne laser and a mode-locked pulsed Ti:Sapphire laser were employed as continuous-wave $(\mathrm{CW})$ and pulsed excitation sources, respectively. The laser beam was focused on one of the mesa structures by employing a x50 objective lens and luminescence was collected through the same lens. The excitation power was controlled by tuning variable neutral-density filter and by monitoring the power in front of the objective lens. Collected luminescence was dispersed by a monochromator and was detected by a Si-chargecoupled device detector or by a streak camera for spectrum measurements and time-resolved measurements, respectively.

Verification of single photon emission was performed with a Hanbury-Brown and Twiss correlation measurement setup [14]. In the present measurements, an exciton emission peak was selected with 0.2-m monochromator. Second-order photon-intensity correlation functions, $\mathrm{g}^{(2)}(\tau)$, were measured under various excitation conditions. Here $\tau$ denotes a delay time between two photon-emission events. Photon antibunching can be evaluated by measuring zero coincidence count $\mathrm{g}^{(2)}(0)=0$, which reflects a probability finding no other photons around a firstly detected photon [15]. Therefore how the value of $g^{(2)}(0)$ is close to zero is a measure of purity for single-photon emission events.

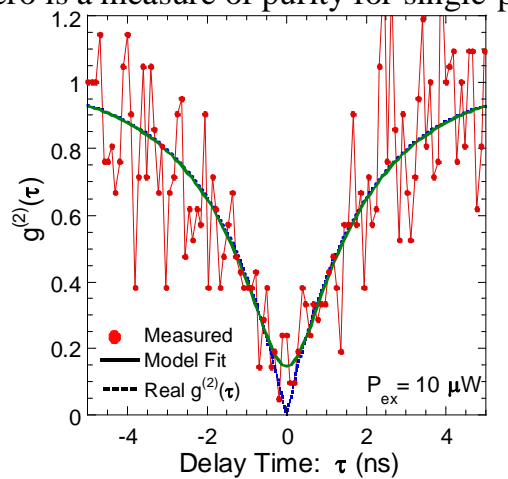

Fig. 1 Measured photon correlation function at $10 \mu \mathrm{W}$ excitation (closed circles) and fitted result (solid line). $\mathrm{g}^{(2)}(\tau)$ (dotted line) is deconvoluted taking into account a time response function of $\exp \left[-(\tau / 0.75)^{2}\right]$.

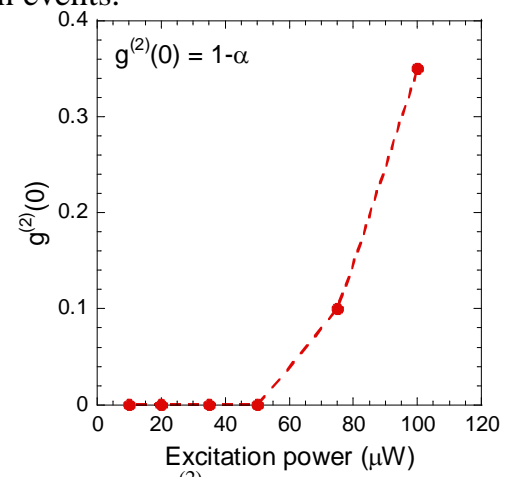

Fig. 2 Real $\mathrm{g}^{(2)}(0)$ value as a function of excitation power.

3 Results Sharp emission line from a single InAlAs QD was observed at the wavelength of 774.4 $\mathrm{nm}$ (photon energy of $1.6009 \mathrm{eV}$ ) as shown in the lower inset in Fig. 3, which coincides well with highly efficient wavelength region of Si-APD. Excitation power dependence of this peak showed linear increase with the 1.1th exponent of the power before saturation at the $\mathrm{CW}$ power of $\sim 150 \mu \mathrm{W}$ (Fig. 4). Therefore this emission line was attributed to an exciton transition (X). Time-Resolved PL measurements were also performed. The recombination lifetime of the exciton peak was found to be $1.13 \mathrm{~ns}$, while a biexciton transition $(2 \mathrm{X})$ located at the lower energy by $\sim 4.6 \mathrm{meV}$ from the exciton line was $0.63 \mathrm{~ns}$. In the following, photon correlation measurements were carried out by selecting the exciton transition line.

Figure 1 shows a measured second-order correlation function under the weak CW excitation power of $10 \mu \mathrm{W}$. Closed circles are the measured data and the solid line is a convoluted curve $f * \mathrm{~g}^{(2)}(\tau)$ fitted to the measured data [16], where $\mathrm{f}$ is time response function of our setup and $\mathrm{g}^{(2)}(\tau)$ is expressed as $\mathrm{g}^{(2)}(\tau)=1$ $\alpha \exp \left[-\left(\mathrm{G}+1 / \tau_{1}\right) \tau\right][17]$. Here, $\alpha$ is a fitting parameter phenomenologically introduced to give a purity of single photon emission, $\mathrm{G}$ is the excitation rate, and $\tau_{1}$ is the exciton lifetime. The dashed line $\mathrm{g}^{(2)}(\tau)$ exhibits a correlation function used in the present fitting procedure and as a result, $\alpha$ is derived to be unity. This is a clear manifestation that highly pure regulated single-photon emission is realized from a single InAlAs QD.

At the higher excitation power, the measured coincidence counts were increased almost linearly with the excitation power as described in ref. 16. However, these measured values do not necessarily reflect 
the true nature of the QD photon emission properties since an influence of measurement setup is included. Instead, coincidence count $\mathrm{g}^{(2)}(0)=1-\alpha$, which is derived from $\mathrm{f} * \mathrm{~g}^{(2)}(\tau)$, can be a measure of the purity of single photon emission. Obtained $g^{(2)}(0)$ was summarized in Fig. 2 as a function of the excitation power. It will be clear that the $g^{(2)}(0)$ values remain almost zero under the weak excitation condition up to the $\mathrm{CW}$ excitation power of $50 \mu \mathrm{W}$. Therefore pure regulated single-photon emission is achieved in this excitation range. In the higher excitation above this critical excitation, however, the $\mathrm{g}^{(2)}(0)$ values are substantially increased and single-photon emission properties were degraded.

4 Discussion In order to explain the observed increase of $g^{(2)}(0)$, the variation of the spectral shape of the exciton peak was closely investigated. The exciton line shape observed at the excitation power of $100 \mu \mathrm{W}$ is shown in the upper inset of Fig. 3. This obviously suggests the presence of an additional peak at the lower energy side of the main peak. This additional peak intensity was estimated by assuming Gaussian lineshape function to give the better overall fitting to the measured spectrum. In Fig. 3, the relative intensity of the additional peak to the main peak is plotted against the excitation power. This excitation-power dependence shown in Fig. 3 shows clear correspondence with the excitation-power dependence of the $g^{(2)}(0)$ value shown in Fig. 2. This strongly suggests that the appearance of the additional peak is closely related to the degradation of the photon anti-bunching properties. Indeed, appearance of additional peak at higher excitation power was predicted for InGaAs by Lomascolo et al. [18]. In this literature, theoretical calculation of optical spectra was carried out under different excitation conditions, and revealed that the transition probability related to a triexciton state will also distribute at the energy region overlapped with the exciton transition. Although the material system is different from our case, however, it is plausible that the spectral overlap of exciton states and multi-exciton states will possibly take place under high excitations.

In order to check the validity of multi-exciton populations, an average number of electron-hole pairs in a single dot was estimated by assuming four-energy-level model depicted in the inset of Fig. 4 [19]. The exciton (biexciton) lifetime of $1.13 \mathrm{~ns}(0.63 \mathrm{~ns})$ and independently measured triexciton lifetime of $0.3 \mathrm{~ns}$ were assumed in this calculation. By solving rate equations for each energy levels, the excitationpower dependence of the steady-state exciton (biexciton) emission intensity $I_{X}\left(I_{2 X}\right)$ were derived as

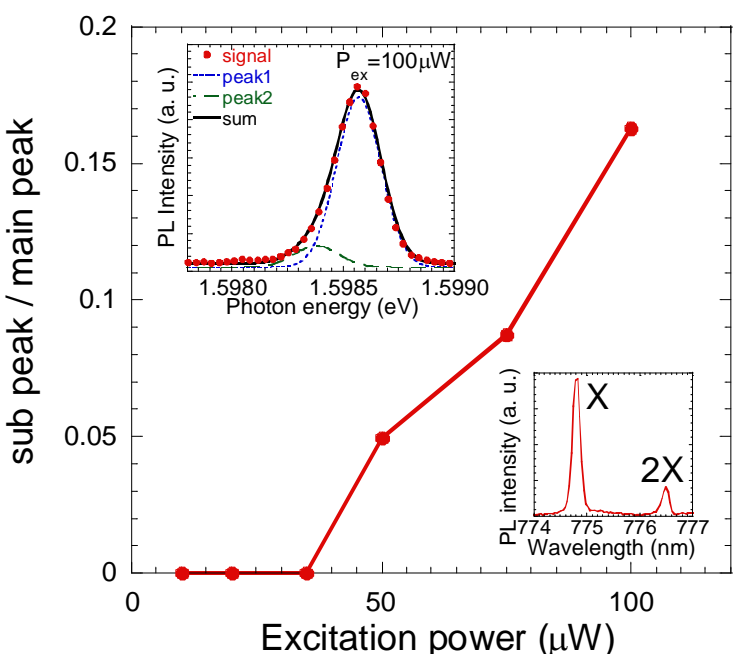

Fig. 3 Relative integrated intensity of additional peak to Fig. 4 Exciton and biexciton peak intensity as a function the main peak as a function of excitation power are of excitation power. Closed circles (squares) are measplotted in the main figure. (upper inset) Exciton line ured $\mathrm{X}(2 \mathrm{X})$ intensities and fitting results with Eq. 1 (Eq. shape at $100 \mu \mathrm{W}$ excitation. Measured data (closed 2) are given by solid (dotted) lines. (inset) Assumed four-

circles), main peak (dashed line), additional peak energy-level model.

(dashed-dot line), and sum of them (solid line). (lower inset) single QD spectrum consisted with exciton peak (X) and biexciton (2X) peak.

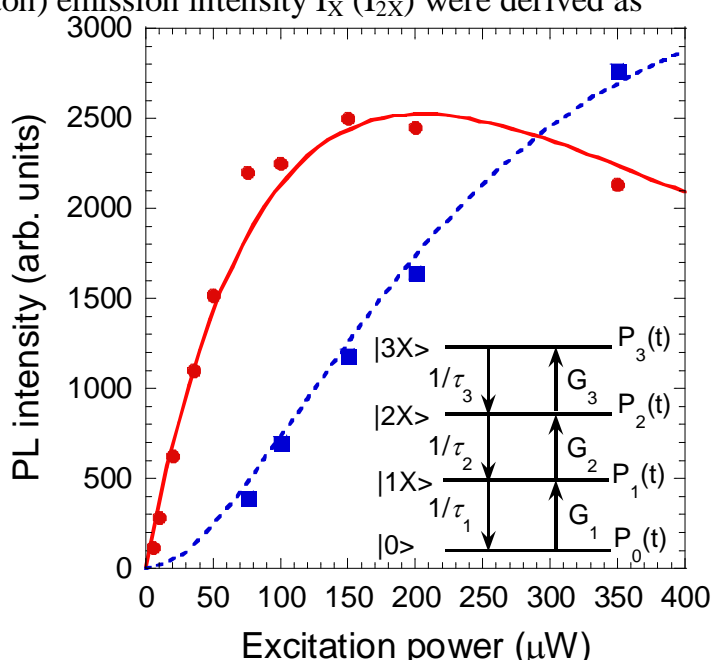




$$
\begin{aligned}
& \mathrm{I}_{\mathrm{X}}(\mathrm{G}) \propto \frac{\mathrm{P}_{1}}{\tau_{1}}=\frac{1}{\tau_{1}}\left(1+\frac{1}{\mathrm{G}_{1} \tau_{1}}+\mathrm{G}_{2} \tau_{2}+\mathrm{G}_{2} \tau_{2} \mathrm{G}_{3} \tau_{3}\right)^{-1}, \\
& \mathrm{I}_{\mathrm{XX}}(\mathrm{G}) \propto \frac{\mathrm{P}_{2}}{\tau_{2}}=\frac{1}{\tau_{2}}\left(1+\frac{1}{\mathrm{G}_{2} \tau_{2}}+\frac{1}{\mathrm{G}_{1} \mathrm{G}_{2} \tau_{1} \tau_{2}}+\mathrm{G}_{3} \tau_{3}\right)^{-1},
\end{aligned}
$$

where $\tau_{\mathrm{i}}$ 's and $\mathrm{P}_{\mathrm{i}}$ 's $(\mathrm{i}=1,2,3)$ are $\mathrm{i}$-exciton lifetimes and probabilities finding i-exciton state in a $\mathrm{QD}$, respectively. As for $G_{i}$, which denotes the excitation rates of $i$-exciton state, $G_{2}$ is assumed to be $1 / 2$ of $G_{1}$ $\left(=\mathrm{G}_{3}\right)$ due to the requirement of spin-selected-excitation based on Pauli's exclusion rule. As shown in Fig. 4 , this model fits very well to the experimental exciton (biexciton) intensities plotted by closed circles (squares). At around $75 \mu \mathrm{W}$, where the additional PL peak became obvious in the measured spectrum, the calculated triexciton intensity or the probability $\mathrm{P}_{3}$ became sizable. Therefore multi-exciton states such as three-exciton or four-exciton states will be involved in this higher excitation range. The excitation of multi-excitons will generate additional photons after the exciton recombination events and this will degrade the purity of single-photon emission at the exciton line. Further qualitative study related to this additional line which emerges under higher excitation is now under way, however, the fact that excitation power dependence of integrated intensity of the additional peak increases with the 1.6th exponent of the power supports the present interpretation.

5 Conclusion Regulated single photon emission from InAlAs QDs was successfully demonstrated at the wavelength within the band where Si-avalanche photodiodes have high quantum efficiencies. Studies of excitation-power dependence of photon coincidence counts revealed that highly pure single-photon emission was maintained up to an onset of multi-exciton contributions. The degradation of the photon anti-bunching was explained with the multi-exciton contributions based on the rate equation analysis.

Acknowledgement This work was supported in part by the Grant-in-Aid for Scientific Research (S) (2), No. 16106005 from the Ministry of Education, Science, Sports, and Culture.

\section{References}

[1] Nicolas Gisin, Grégoire Ribordy, Wolfgang Tittel, and Hugo Zbinden, Rev. Mod. Phys. 74 (2002) 145.

[2] N. Gisin, G. Ribordy, W. Tittel, and H. Zbinden, Rev. Mod. Phys. 74 (2002) 145.

[3] For a review, C. Santori, D. Fattal, J. Vuckovic, G. S Solomon and Y. Yamamoto, New J. Phys. 6 (2004) 89.

[4] E. Moreau, I. Robert, J. M. Gérard, I. Abram, L. Manin, and V. Thierry-Mieg. Appl. Phys. Lett. 79 (2001) 2865.

[5] A. Kiraz, P. Michler, C. Becher, B. Gayral, A. Imamoglu, L. Zhang, E. Hu, W. V. Schoenfeld, and P. M. Pertroff, Phys. Rev. B 63 (2001) 121312.

[6] C. Santori, M. Pelton, G. Solomon, Y. Dale, and Y. Yamamoto, Phys. Rev. Lett. 86 (2001) 1502.

[7] V. Zwiller, T. Aichele, W. Seifert, J. Persson, and O. Benson, Appl. Phys. Lett. 82 (2003) 1509.

[8] X. Brokmann, E. Giacobino, M. Dahan, and J. P. Hermier, Appl. Phys. Lett. 85 (2004) 712.

[9] C. Couteau, S. Moehl, F. Tinjod, J. M. Gérard, K. Kheng, H. Mariette, J. A. Gaj, R. Romestain, and J. P. Poizat, Appl. Phys. Lett. 85 (2004) 6251.

[10] Charles Santori, Stephan Gözinger, and Yoshihisa Yamamoto,Satoshi Kako, Katsuyuki Hoshino, and Yasuhiko Arakawa, Appl. Phys. Lett. 87 (2005) 051916.

[11] K. Takemoto, Y. Sakuma, S. Hirose, T. Usuki, N. Yokoyama, T. Miyazawa, M. Takatsu, and Y. Arakawa, Jpn. J. Appl. Phys. 43 (2004) 993.

[12] T. Miyazawa, K. Takemoto, Y. Sakuma, S. Hirose, T. Usuki, N. Yokoyama, M.Takatsu, and Y. Arakawa, Jpn. J. Appl. Phys. 44 (2005) L620.

[13] T. Yokoi, S. Adachi, H. Sasakura, S. Muto, H. Z. Song, T. Usuki, and S. Hirose, Phys. Rev. B 71 (2005) 041307.

[14] R. Hanbury-Brown and R. Q. Twiss, Nature 177 (1956) 27.

[15] R. Loudon, The Quantum Theory of Light (Oxford University Press. Oxford, New York, 2000) 3rd ed, chapter 9.

[16] S. Kimura, H. Kumano, M. Endo, I. Suemune, T. Yokoi, H. Sasakura, S. Adachi, S. Muto, H. Z. Song, S. Hirose, and T. Usuki, Jpn. J. Appl. Phys. 44 (2005) L793.

[17] B. Lounis, H. A. Bechtel, D. Gerion, P. Alivisatos and W. E. Moerner, Chem. Phys. Lett. 329 (2000) 399.

[18] M. Lomascolo, A. Vergine, T. K. Johal, R. Rinaldi, A. Passaseo, R. Cingolani, S. Patane, M. Labardi, M. Allegrini, F. Troiani, and E. Molinari, Phys. Rev. B 66 (2002) 041302.

[19] D.V. Regelman, U. Mizrahi, D. Gershoni, and E. Ehrenfreund, Phys. Rev. Lett. 87 (2001) 257401.

(C) 2004 WILEY-VCH Verlag GmbH \& Co. KGaA, Weinheim 